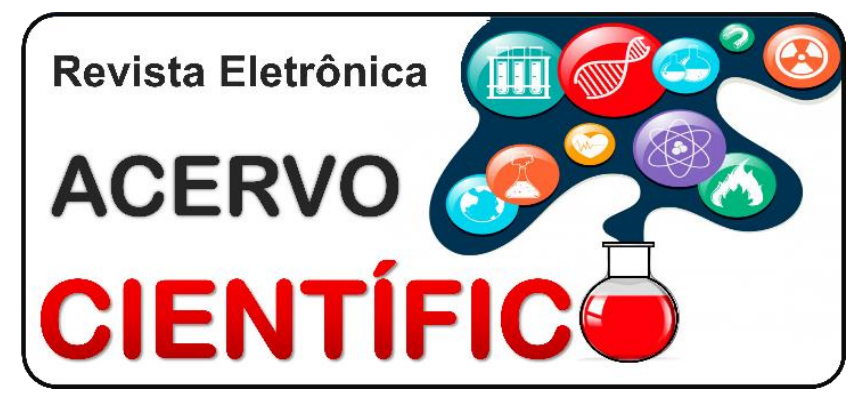

ARTIGO ORIGINAL

Recebido em: 1/2020

Aceito em: 2/2020

Publicado em: 6/2020

\title{
Promoção da adesão ao tratamento de hipertensão arterial sistêmica e diabetes mellitus em uma estratégia saúde da família
}

\author{
Promotion of adherence to the treatment of systemic arterial hypertension and diabetes \\ mellitus in a family health strategy
}
Promoción de la adherencia al tratamiento de la hipertensión arterial sistémica y la diabetes mellitus en uma estrategia de salud familiar

Rodrigo Batista da Silva ${ }^{1 *}$, Adriel Raiol Aragão, Ana Luiza Sousa Barbosa1, Danilo Rocha de Aguiar $^{1}$, Patrícia Oliveira Bezerra ${ }^{1}$, Paula Yasmin Camilo Coelho ${ }^{1}$, Renato da Silva Pujadas ${ }^{1}$, Érica Furtado Azevedo Coelho'.

\begin{abstract}
Resumo: O objetivo da presente pesquisa foi avaliar a não adesão ao tratamento de hipertensão arterial sistêmica e diabetes mellitus de pacientes e realizar intervenção em saúde. Foi um estudo de caráter intervencionista buscou promover a adesão ao tratamento de Hipertensão Arterial Sistêmica (HAS) e Diabetes Mellitus (DM) dos pacientes atendidos na Estratégia de Saúde da Família (ESF) em uma cidade do Pará, sem nenhum financiamento externo, aprovado no Comitê de Ética em Pesquisa (CEP) avaliando informações sobre antes e após uma intervenção de educação em saúde sobre a temática para os sujeitos da pesquisa. A maioria dos pacientes não esquecem de tomar a medicação, não ingerem frutas e verduras na dieta e se sentem bem tratados na ESF que fazem acompanhamento. A adesão ao tratamento e os autocuidados com alimentação são de fundamental importância para evitar complicações. Com isso, percebe-se a necessidade da ocorrência de novas intervenções e promoções de saúde nas Unidades Básicas de Saúde para obter uma maior adesão ao tratamento medicamentoso, aumento da prática de atividade física e aumento na alimentação do consumo de verduras e frutas.
\end{abstract}

Palavras-chave: Hipertensão, Diabetes mellitus, Terapia.

Abstract: The objective of the present research was to evaluate non-adherence to the treatment of systemic
arterial hypertension and diabetes mellitus in patients and to carry out health intervention. It was an
interventionist study that sought to promote adherence to the treatment of Systemic Arterial Hypertension
(SAH) and Diabetes Mellitus (DM) of patients seen in the Family Health Strategy (FHS) in a city in Pará,
without any external funding, approved by the Research Ethics Committee (CEP) evaluating information on
before and after a health education intervention on the subject for the research subjects. Most patients do
not forget to take their medication, do not eat fruits and vegetables in the diet and feel well treated in the
FHS who are followed up. Adherence to treatment and self-care with food are of fundamental importance to
avoid complications. As a result, there is a need for new health interventions and promotions in Basic Health

${ }^{1}$ Centro Universitário Metropolitano da Amazônia (UNIFAMAZ), Belém - Pará.

*E-mail: rodrigo.batista.14@hotmail.com 
Units to achieve greater adherence to drug treatment, increased physical activity and increased consumption of vegetables and fruits.

Keywords: Hypertension, Diabetes mellitus, Therapy.

Resumen: El objetivo de la presente investigación fue evaluar la no adherencia al tratamiento de la hipertensión arterial sistémica y la diabetes mellitus en pacientes y llevar a cabo una intervención sanitaria. Fue un estudio intervencionista que buscaba promover la adherencia al tratamiento de la hipertensión arterial sistémica (HSA) y la diabetes mellitus (DM) de pacientes atendidos en la Estrategia de salud familiar (FHS) en una ciudad en Pará, sin ningún financiamiento externo, aprobado por el Comité de Ética de Investigación (CEP) que evalúa información sobre antes y después de una intervención de educación sanitaria sobre el tema para los sujetos de investigación. La mayoría de los pacientes no se olvidan de tomar sus medicamentos, no comen frutas y verduras en la dieta y se sienten bien tratados en la FHS que reciben seguimiento. La adherencia al tratamiento y el autocuidado con los alimentos son de fundamental importancia para evitar complicaciones. Como resultado, existe la necesidad de nuevas intervenciones y promociones de salud en las Unidades Básicas de Salud para lograr una mayor adherencia al tratamiento farmacológico, una mayor actividad física y un mayor consumo de verduras y frutas.

Palabras clave: Hipertensión, Diabetes mellitus, Terapia.

\section{INTRODUÇÃO}

A população brasileira desde a metade do século passado sofreu intensas transformações sociais, econômicas, políticas e culturais, o que inclui no seu perfil epidemiológico o aumento das taxas de morbimortalidade por problemas crônicos de saúde que alteram a qualidade de vida e de saúde das pessoas (MACIEL EAM, 2012). Dentre esses problemas a Hipertensão arterial sistêmica (HAS) e o Diabetes mellitus (DM) representam dois dos principais fatores de risco, contribuindo decisivamente para o agravamento deste cenário em nível nacional devendo ser tratados primariamente na Estratégia Saúde da Família (ESF), haja vista ser o contato mais próximo da população do que consultas especializadas ou hospitais (BRASIL, 2009).

A HAS, no mundo é uma das doenças que mais atinge a população (com até $15-20 \%$ da população adulta ou idosa), já no Brasil, esses dados são corroborados pois $20 \%$ dos adultos (sem distinção de sexo, idade ou raça) são hipertensos com maior prevalência na região urbana das cidades do Sudeste e Sul em comparação com as outras regiões do país. É uma condição clínica multifatorial definida por níveis cronicamente elevados da pressão arterial - PA (PA $\geq 140 \times 90 \mathrm{mmHg})$. É caracterizada por ter alta prevalência e baixo controle, e por isso, constitui um problema de saúde pública de grande relevância, pois reduz a expectativa de vida e conduz a diversas complicações cardíacas e renais, além de gerar elevado custo econômico e social (OSHIRO ML, 2007; MINISTÉRIO DA SAÚDE, 2013). Cerca de 40 a 83\% da população hipertensa desconhece o seu diagnóstico, sendo que de 75 a $92 \%$ daqueles que estão em tratamento não controlam a pressão arterial (PA) (OSHIRO ML, 2007; DOSSE C, 2009).

Estudos demonstram que, para hipertensos, o abandono do tratamento (feito com medicações que abaixam a pressão arterial sistêmica) é grande, conforme o tempo decorrido após o início da terapêutica. Vários fatores influenciam na adesão ao tratamento e podem estar relacionados ao paciente (idade, sexo, estado civil, etnia, nível socioeconômico e escolaridade); à doença (ausência de sintomas, cronicidade, consequências tardias); aos hábitos de vida, crenças de saúde e hábitos culturais (percepção da seriedade do problema, desconhecimento, experiência com a doença no contexto familiar e autoestima); ao tratamento dentro do qual se engloba a qualidade de vida (custo, efeitos indesejáveis, esquemas terapêuticos complexos); à instituição (política de saúde, acesso ao serviço de saúde, tempo de espera versus tempo de atendimento); e, finalmente, ao relacionamento com a equipe de saúde (LIMA TM, et al., 2010). 
Já a DM possui prevalência de até $7,5 \%$ dos adultos brasileiros principalmente em ambientes urbanos de grandes cidades. É um transtorno metabólico de multiplica causas, definida por hiperglicemia e distúrbios no metabolismo de carboidratos, proteínas e gorduras, resultantes de defeitos da secreção e/ou da ação da insulina, sendo este, outro grande problema da saúde brasileira devido ao amento da sua prevalência e por estar associada a complicações (MINISTÉRIO DA SAÚDE, 2013). As duas patologias são consideradas um problema sensível a Atenção Primaria, pois o bom manejo clínico evita hospitalizações e mortes por complicações cardiovasculares e cerebrovasculares (SOUZA TP, et al., 2015). Essa doença é diagnosticada com exame de sangue chamado Glicemia em Jejum ou Hemoglobina Glicada, além de uma opção chamada Teste de Tolerância Oral à Glicose com valores específicos de diagnóstico de diabetes para cada exame.

O diabetes mellitus constitui um desafio e problema para portadores, suas famílias e profissionais de saúde para a obtenção de um bom e rigoroso controle glicêmico e metabólico, a fim de minimizar complicações em curto e longo prazo da doença. Mudanças comportamentais (como atividade física regular e alimentação saudável) e adesão ao tratamento medicamentoso são essenciais para prevenção das complicações agudas e crônicas. O profissional deve entender cada contexto de paciente, negociar prioridades, monitorar a adesão, motivar a participação no tratamento e reforçar o esforço do paciente no manejo do autocuidado. A não adesão ao tratamento é um problema conhecido no cenário nacional e internacional, pois prejudica a resposta fisiológica à doença e gera a possibilidade de ter mais complicações, a relação profissional-paciente, aumenta o custo direto e indireto do tratamento (FARIA VB, 2013; LADEIA HT, 2013; ADA, 2013)

Mesmo sendo sensível a Atenção primaria, a HAS e a DM são responsáveis pela primeira causa de hospitalização e de morte no Brasil, sendo a falta de adesão ao tratamento um dos principais fatores desencadeadores das complicações dessas patologias. Esse fato acontece pelo caráter silencioso dessas patologias, a qual cerca de $30 \%$ da população acometida pela doença não sabe que a possui ou não faz 0 tratamento corretamente por falta de motivação ou recursos (SANTOS ZMSA, et al., 2009; ANDRADE TL, 2010; ALVES BA, et al., 2012).

Assim, para o sucesso da adesão, o indivíduo, ao iniciar o tratamento, deve receber orientações suficientes sobre a funcionalidade da medicação prescrita, os efeitos colaterais, a frequência de ingestão, a quantidade diária e possíveis interações medicamentosas. Contudo, muitos pacientes não recebem informações adequadas vindas dos profissionais da saúde sobre 0 as características do tratamento medicamentoso, muitas vezes esquecem de informar que os efeitos destes medicamentos podem influenciar no cotidiano do paciente, já que durante o processo podem ocorrer efeitos colaterais indesejados (náuseas, vômitos e tonturas) (ANDRADE DMC, et al., 2011; MILECH A, 2016).

Para tanto, o objetivo do presente artigo foi promover a adesão ao tratamento de Hipertensão Arterial Sistêmica e Diabetes Mellitus dos pacientes atendidas pela Estratégia Saúde da Família (ESF) em uma cidade do Pará.

\section{MÉTODOS}

Foi um estudo de caráter intervencionista buscou promover a adesão ao tratamento de HAS e DM dos pacientes atendidos na Estratégia de Saúde da Família (ESF) em uma cidade do Pará, sem nenhum financiamento externo. Os pacientes incluídos neste projeto foram estudados segundo os preceitos da Declaração de Helsinque e do Código de Nuremberg, seguindo as determinações da Resolução 466/2012 do Conselho Nacional de Saúde, sendo avaliado e aprovado pelo Comitê de Ética e Pesquisa da Faculdade Metropolitana da Amazônia - FAMAZ, cumprindo-se as orientações descritas no Termo de Consentimento Livre e Esclarecidas-TCLE, e após o aceite do orientador, da instituição, da Estratégia Saúde e do Comitê de Ética em Pesquisa (CEP) sob CAAE 80669617.8.0000.5701.

A população alvo do presente estudo foram os pacientes atendidos pela Estratégia de Saúde da Família, que não aderiram o tratamento de HAS e DM. Foram incluídos no Projeto de Intervenção todos os pacientes com HAS e/ou DM que não aderiram ao tratamento, acima de 50 anos de idade, residentes da área coberta 
pela ESF e que voluntariamente concordaram em participar do estudo, mediante assinatura do Termo de Consentimento Livre e Esclarecido (TCLE). Foram excluídos do Projeto todos os pacientes que não tinham comorbidades, idade inferior a 50 anos, não residentes da área coberta pela ESF e que não aceitaram participar das atividades.

Palestras socioeducativas, atividades lúdicas e visitas domiciliares. Dentre as atividades destinadas aos pacientes foram empregadas vivencias em grupo, jogos educativos e peças de teatro. Questionário que avaliou o conhecimento dos autocuidados relacionados a complicações de HAS e DM. Foram empregadas entrevistas para elaboração de relatórios e aplicação dos questionários, para atentar os pacientes sobre o projeto, sendo utilizados cartazes informativos e distribuição de folhetos, sendo passado para os pacientes antes e após a intervenção.

Os instrumentos de coleta de dados utilizados foram questionários aplicados pelos próprios examinadores em pacientes hipertensos e/ou diabéticos cadastrados na Estratégia Saúde da Família Palmeira do Açaí. O questionário, construído pelos autores, sobre Hipertensão avaliou se o participante adere ou não ao tratamento com itens validados por especialistas que constituem uma gama de características discutidas na literatura e nas instituições de saúde associadas ao processo de não adesão à terapêutica, obtendo o Instituto Verificador de Circulação (IVC) de amplitude de 1,0 sendo assim, consistente e válido. Já o questionário sobre Diabetes se o participante possuía atividades de autocuidado com essa doença, sendo este questionário validado pela Sociedade Brasileira de Endocrinologia (BRASIL, 2009).

Para a confecção de dados e tabelas foi utilizado o software Excel 2016, e para a redação do texto o Word 2016. De acordo com a natureza das variáveis, foi aplicada análise estatística descritiva e comparativa sendo informados os valores percentuais dos dados analisados.

\section{RESULTADOS}

Foram coletados dados com 13 sujeitos de pesquisa sendo 5 do sexo masculino e 8 do sexo feminino, com média de idade de 55 anos (84,6\%), 53\% possuem primeiro grau incompleto, $61,5 \%$ casado (a). Foi avaliado a não adesão ao tratamento de hipertensão arterial sistêmica com foco no uso de medicação. Não houve significância estatística com o teste qui-quadrado para as cinco perguntas formuladas, contudo é possível destacar um aumento no número de pessoas que assinalaram "nunca ou quase nunca" para a Pergunta 1 (quando está bem, deixa de tomar a medicação?), Pergunta 4 (faltou as consultas para acompanhamento da doença?) e Pergunta 5 (para de tomar a medicação se sentir mal?) após a intervenção dos autores (Figura 1). 
Figura 1 - Representação gráfica da avaliação da não adesão ao tratamento de pressão arterial: uso da medicação e tratamento antes e após a intervenção dos pesquisadores.

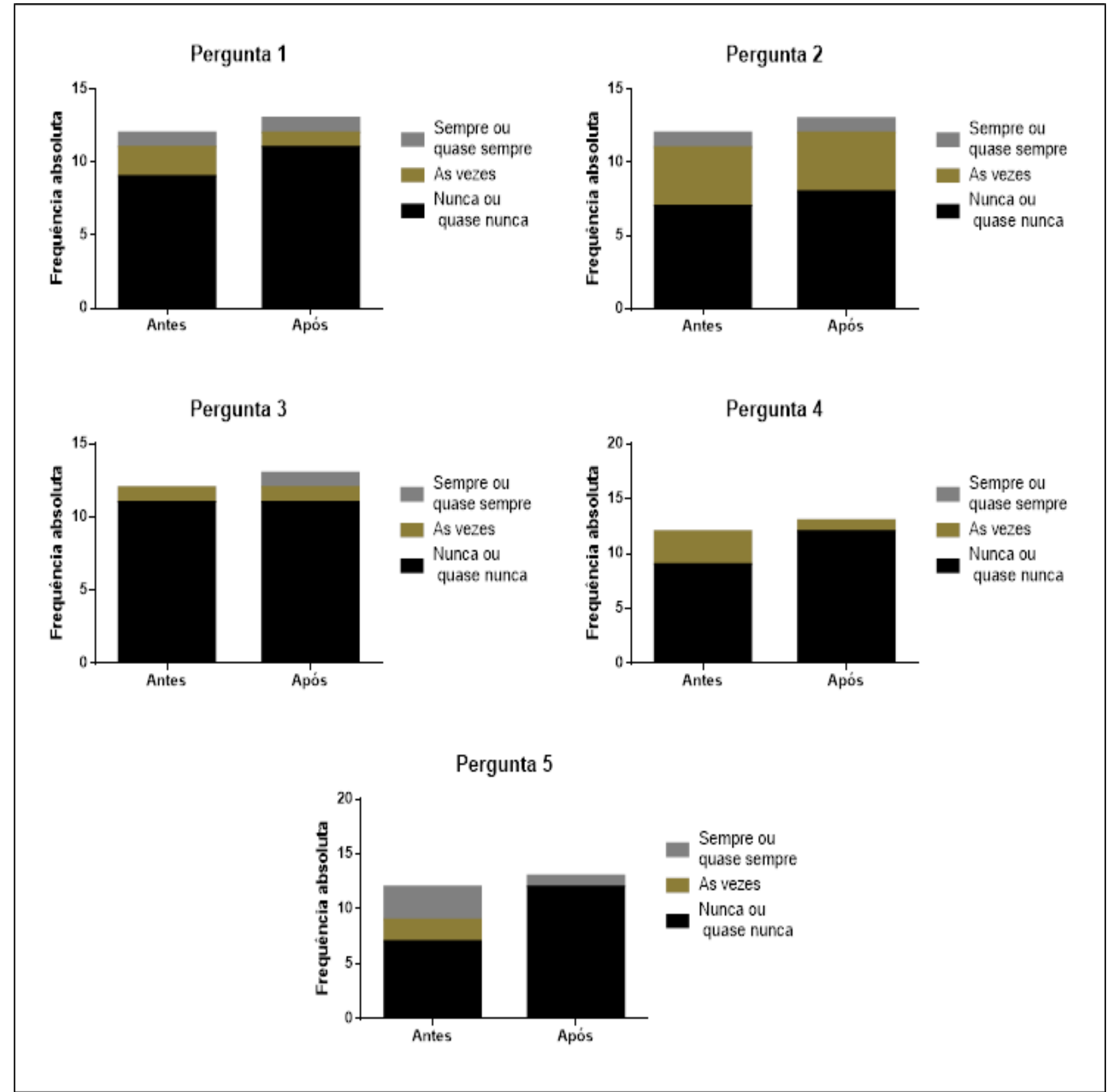

Legenda: Pergunta 1: Quando está bem, deixa de tomar a medicação? ( $p=0,7811)$. Pergunta 2: Esqueceu de tomar o medicamento alguma vez? $(p=0,9867)$. Pergunta 3: Deixa de tomar a medicação quando a unidade de saúde não oferece? $(p=0,6183)$. Pergunta 4: Falta as consultas para acompanhamento de hipertensão? $(p=0,4989)$. Pergunta 5: Para de tomar a medicação por sentir-se mal com ela? $(p=0,1173)$. Teste qui-quadrado. Fonte: Silva RB, et al., 2020.

Após isso, foi avaliado a alimentação de pessoas com hipertensão arterial sistêmica dos sujeitos da pesquisa. Quando questionados sobre o consumo de frutas e verduras, a maioria dos participantes afirmaram consumir "as vezes" ou "quase nunca" e, este resultado, teve significância estatística $(p=0,0091)$.

Foi avaliado questões gerais de saúde dos pacientes que realizam tratamento de hipertensão arterial sistêmica. Quando perguntado se se sentia mal quando atendido na unidade de saúde que faz acompanhamento, a maioria disse que nunca ou quase nunca, sendo este um valor significativamente estatístico $\mathrm{p}=0,0339$ (Figura 3). 
Figura 2 - Representação gráfica da avaliação da não adesão ao tratamento de pressão arterial: alimentação antes e após a intervenção dos pesquisadores.

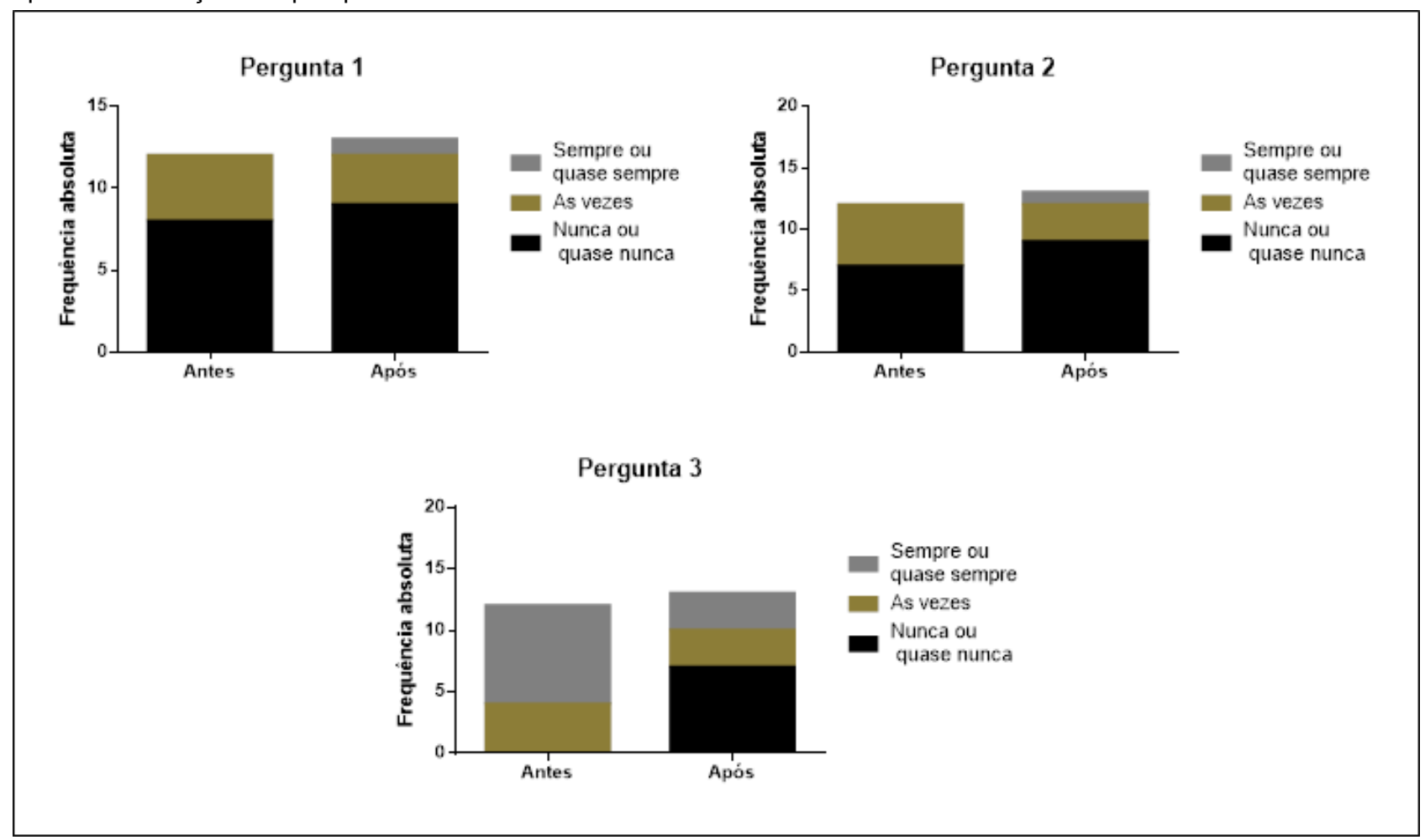

Legenda: Pergunta 1: Com o início do tratamento, faz uso de alimentos ricos em sal? ( $p=0,5589)$. Pergunta 2: Com o início do tratamento, fez uso de alimentos ricos em gordura? ( $p=0,4247)$. Pergunta 3: Possui alimentação pobre em frutas e verduras? $\left(p=0,0091^{\star}\right)$. Fonte: Silva RB, et al., 2020.

Figura 3 - Representação gráfica da avaliação da não adesão ao tratamento de pressão arterial: questões gerais de saúde, antes e após a intervenção dos pesquisadores.

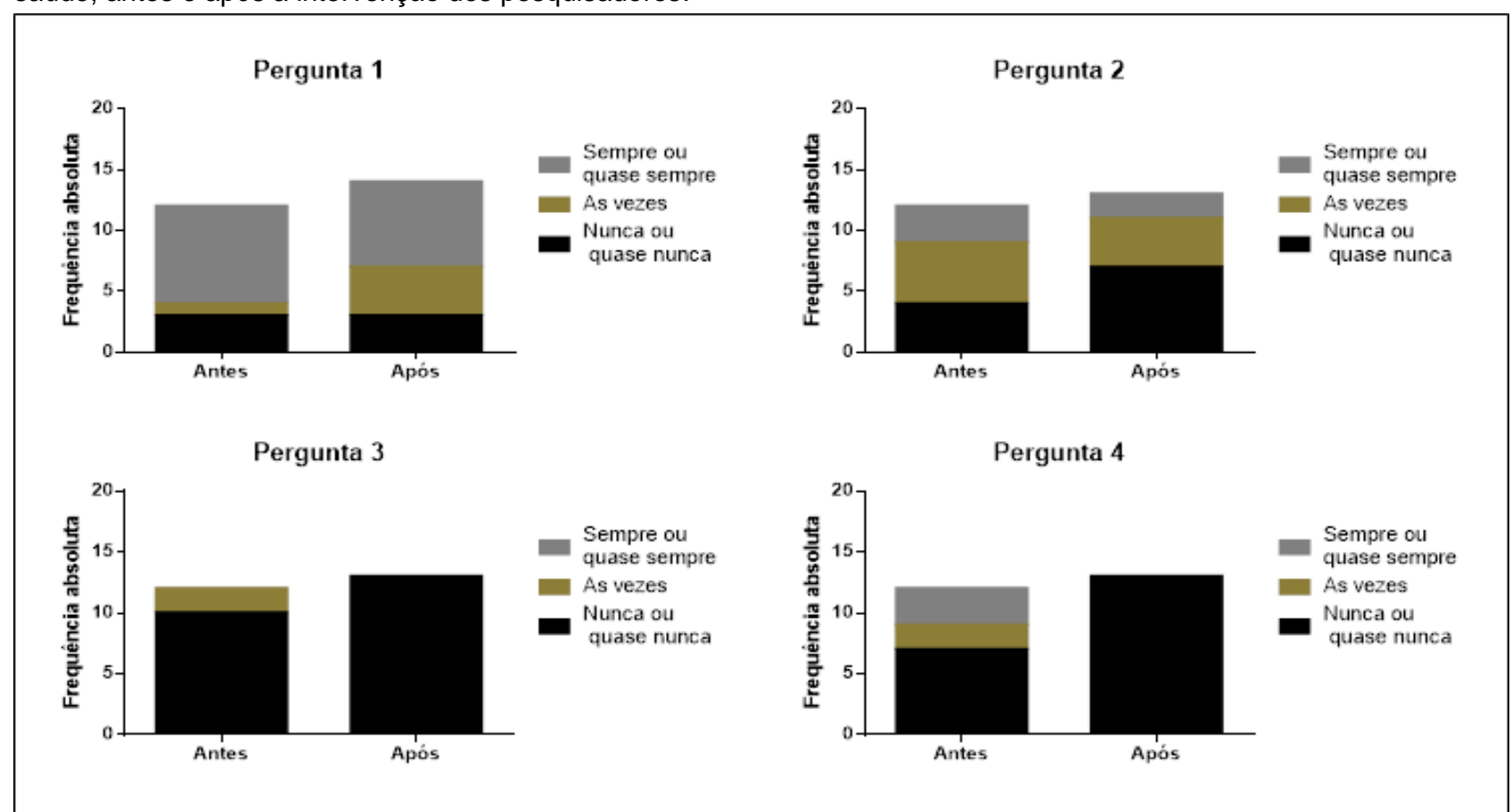

Legenda: Pergunta 1: Procura com frequência a unidade de saúde porque sua pressão arterial está alta? $(p=0,4225)$. Pergunta 2: Quando aferida sua pressão arterial, geralmente encontra-se elevada? $(p=0,5795)$. Pergunta 3: Sente dificuldades em conversar com os profissionais de saúde? $(p=0,3080)$. Pergunta 4: Sente-se mal atendido na unidade de saúde que faz acompanhamento? $\left(p=0,0339^{\star}\right)$. Teste qui-quadrado. Fonte: Silva RB, et al., 2020. 
Foi avaliado a monitoração da glicemia, atividade física e medicação dos pacientes diabéticos. Quando perguntado em quantos dos últimos sete dias tomou as medicações conforme orientado, a maioria disse que em 6 ou 7 dias por semanas, sendo este um valor significativamente estatístico $(p=0,0266)$ (Figura 4).

Figura 4 - Representação gráfica do questionário de atividades de autocuidado com o diabetes: monitoração da glicemia, atividade física e medicação.

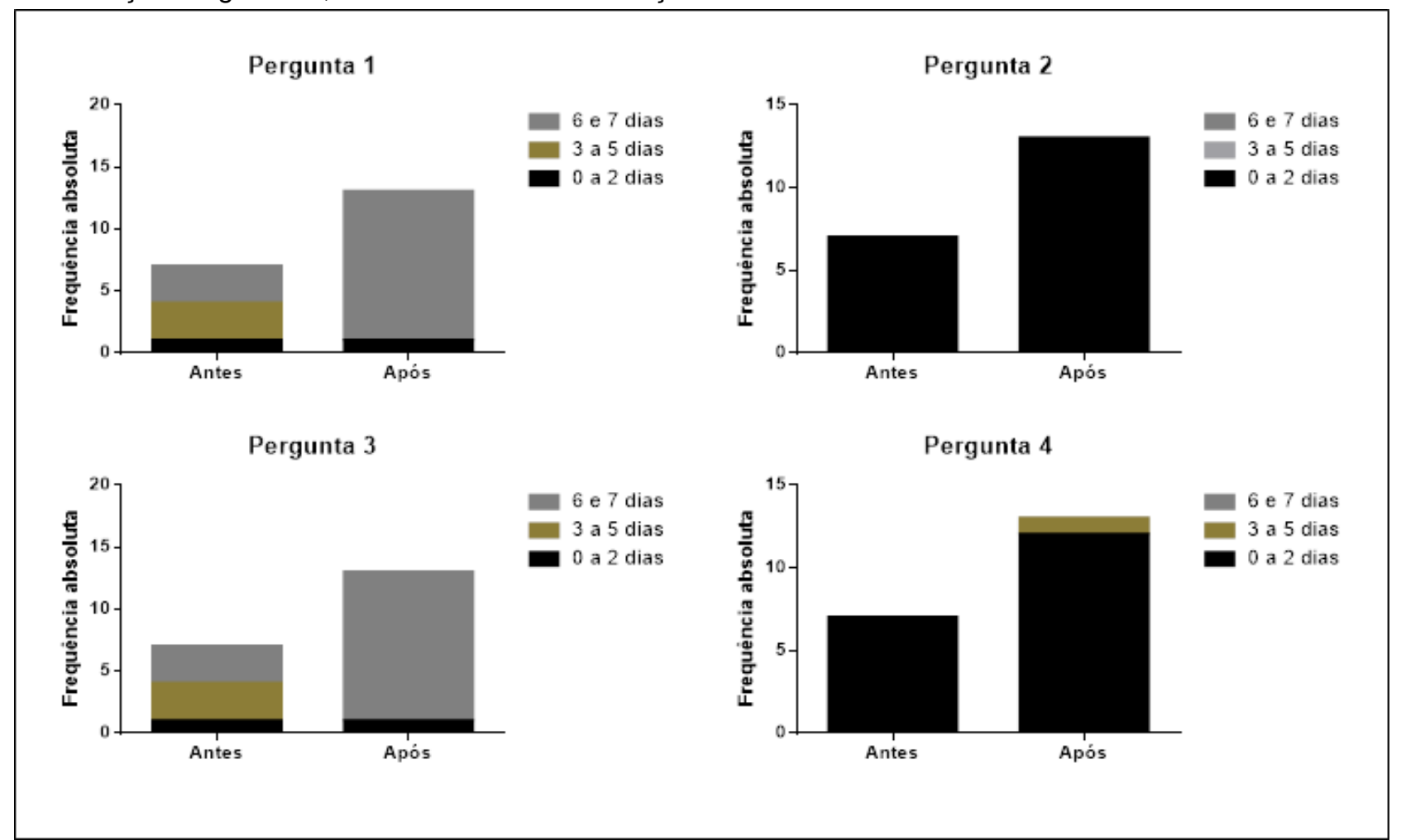

Legenda: Pergunta 1: Em quantos dos últimos sete dias tomou seus medicamentos do diabetes conforme recomendação? $\left(p=0,0266^{*}\right)$. Pergunta 2: Em quantos dos últimos sete dias tomou suas injeções de insulina conforme recomendado? ( $p$ não analisado). Pergunta 3: Em quantos dos últimos sete dias tomou o número indicado de comprimidos de diabetes? $(p=0,0266)$. Pergunta 4 : Em quantos dos últimos sete dias avaliou a glicemia? ( $p=0,7)$. Teste qui-quadrado. Fonte: Silva RB, et al., 2020.

O presente estudo foi realizado com uma população constituída de treze indivíduos, após terem sido aplicados os critérios de exclusão propostos pelo trabalho. Sendo assim, o número de indivíduos do presente estudo foi de cinco pacientes do sexo masculino e oito do sexo feminino, tendo como a idade predominante mais de sessenta anos, com o primeiro grau incompleto e com o estado civil de casado.

É pertinente destacar antes de adentrar na discussão as limitações encontradas no decorrer da pesquisa, que não foram previstas antes da coleta de dados, como a não adesão à participação da proposta de intervenção e o não preenchimento de todos os itens dos questionários que foram aplicados após a intervenção. Assim, acarretou uma maior disposição de tempo dos pesquisadores para que conseguissem efetuar a coleta de dados de forma correta visando minimizar algum possível comprometimento da pesquisa.

\section{DISCUSSÃO}

O Diabetes Mellitus e a Hipertensão Arterial Sistêmica possuem uma alta morbimortalidade relacionada a não adesão de muitos dos portadores dessas patologias ao tratamento, dessa forma, tornam-se um problema de saúde pública que compromete a produtividade, qualidade de vida e sobrevida dos indivíduos. (SILVA MCM, et al., 2013). 
Nesse sentido, esta pesquisa propõe além de promover a adesão ao tratamento de hipertensos e diabéticos da atenção básica escolhida, encontrar a melhor forma de promover a educação em saúde desta população pesquisada para servir de exemplo e bibliografia para outras pesquisas semelhantes melhorarem a educação em saúde, diminuírem custos e verbas advindos do governo e aumentar a eficácia de pesquisa que possuam tais objetivos.

A priori, no início do estudo, os resultados esperados eram que maioria dos pacientes diabéticos e hipertensos tivesse uma adesão ao tratamento precária, tendo como agravantes o não uso contínuo da medicação e alimentação inadequada. Contudo, na presente pesquisa foi obtido como resultado inicial uma maior prevalência de indivíduos que se declararam serem mais conscientes em relação à alimentação referente ao uso de sal no preparo de alimentos e a ingesta de alimentos ricos em carboidratos.

Essa realidade encontra variações regionais e de localidade de acordo com o conhecimento de si próprio como ser biopsicossocial, na relação médico paciente e na associação saúde-doença, devendo encontrar apoio em estatísticas que analisam que quanto maior o nível socioeconômico ou educacional, maior conhecimento sobre a doença o paciente possui e, com isso, maior adesão ao tratamento e diminuição da possibilidade de consequências das doenças Hipertensão Arterial Sistêmica ou Diabetes Mellitus (REZENDE DS, et al., 2015).

Todavia a presente pesquisa identificou que apesar dos pacientes já possuírem uma consciência da minimização do uso de sal no preparo da comida e da ingestão de alimentados hipercalóricos, os mesmos tinham uma alimentação pobre em frutas e verduras. Observou-se que após a intervenção realizada na Unidade de Atenção Básica houve uma maior prevalência de indivíduos que faziam uso de uma alimentação com verduras e frutas e essa análise é importante haja vista, diminuir a possibilidade de ingestão de alimentos saturados ou rico em carboidratos, o que pode prejudicar ou até mesmo agravar a doença de Diabetes Mellitus ou Hipertensão Arterial Sistêmica.

Segundo CARVALHO (2012), o consumo de alimentos com baixos índices glicêmicos e ricos em fibras alimentares induz menor aumento nos níveis séricos de glicose e insulina no período pós-prandial. Assim, as orientações nutricionais, são consideradas essenciais para o controle do Diabete Mellitus (DM) e da Hipertensão arterial (HAS). Portanto, a presente promoção de adesão ao tratamento demonstrou ter associação positiva em relação ao maior consumo de frutas e verduras e esta realização na presente pesquisa pode servir de bibliografia para diferentes pesquisas que encontram a educação em saúde como fator promotor do conhecimento e, consequentemente, da melhoria do bem-estar dos pacientes com essas doenças.

Observou-se que após a promoção de adesão ao tratamento não houve associação entre a intervenção e o estilo de vida. Nesse quesito, talvez devesse ter sido dado mais ênfase ou fossem necessárias mais intervenções para conscientização de tal atividade como fundamental no tratamento e no controle da DM e da HAS; pois além da redução da pressão arterial (PA) pós-exercício e está associado à diminuição dos fatores de risco cardiovasculares e à menor morbimortalidade.

Ademais neste estudo, observou uma maior frequência do uso de medicamentos do diabetes conforme foi recomendado pelo médico durante os últimos sete dias, quando comparados com os dados antes da intervenção realizada já havia descrito uma tendência a de melhores taxas de adesão para o tratamento medicamentoso, e piores taxas de adesão para as atividades de cuidado, tais como, a prática de exercícios físicos e essa realidade pode melhorar, ao longo do tempo a adesão ao tratamento e diminuir riscos de consequências da diabetes, tais como o pé diabético (SOCIEDADE BRASILEIRA DE CARDIOLOGIA, 2010; REZENDE DS, et al., 2015),

\section{CONCLUSÃO}

Por meio deste estudo, foi possível encontrar que a população de pacientes se sente bem tratado na ESF que realiza acompanhamento, normalmente não esquece de tomar medicações se for bem orientado e não utilizada frutas e legumes na dieta normal. Esses dados são importantes para gerar palestras de 
educação em saúde direcionadas para as principais problemáticas da população estudada (alimentação). A Diabetes Mellitus e a Hipertensão Arterial sistêmica são doenças crônicas de grande morbimortalidade na população. A adesão ao tratamento e os autocuidados são de fundamental importância para evitar complicações. Com isso, percebe-se a necessidade da ocorrência de novas intervenções e promoções de saúde nas Unidades Básicas de Saúde para obter uma maior adesão ao tratamento medicamentoso, aumento da prática de atividade física e aumento na alimentação do consumo de verduras e frutas. Sendo necessário o repasse a população de informações acerca da Diabetes e Hipertensão, com o intuito de esclarecer as dúvidas sobre as condutas mais adequadas para se atingir uma estabilidade do quadro clínico das patologias e melhorar qualidade de vida desses indivíduos.

\section{REFERÊNCIAS}

1. AMERICAN DIABETES ASSOCIATION (ADA). Standards Of Medical Care In Diabetes- 2013.Diabetes Care, Alexandria, 2013; 36(Suppl. 1).

2. ALVES BA, et al. Aspectos Determinantes Da Adesão Ao Tratamento De Hipertensão E Diabetes Em Uma Unidade Básica De Saúde Do Interior Paulista. Curso De Enfermagem Da Universidade Paulista, Campinas-SP, Brasil, Health Sci Inst. 2012; 30(3):255-60.

3. ANDRADE TL. Caraterização Da Associação Entre Diabetes Mellitus E Hipertensão Arterial Primaria: Estudo Quantitativo No Município De Jeceaba-Mg, Belo Horizonte, Minas Gerais, Trabalho de Conclusão de Curso. 2010.

4. ANDRADE DMC, et al. Adherence To The Treatment Of Diabetes Mellitus And Systemic Arterial Hypertension: A Focus On Gender Relations Rev Enferm Ufpe On Line. 2011, 5(10): 20-34.

5. BRASIL. Ministério Da Saúde (BR), Departamento De Atenção Básica. Coordenação Nacional De Hipertensão E Diabetes. Secretaria De Atenção À Saúde, Jan, 2009.

6. BRASIL. Ministério Da Saúde (BR), Secretaria De Atenção À Saúde, Hipertensão Arterial Sistêmica Estratégias Para O Cuidado Da Pessoa Com Doença Crônica, Caderno De Atenção Básica N³7. Brasilia, 2013

7. BRASIL. Ministério Da Saúde (BR), Secretaria De Atenção À Saúde, Diabetes Mellitus Estratégias Para O Cuidado Da Pessoa Com Doença Crônica, Caderno De Atenção Básica №36. Brasilia, 2013.

8. CARVALHO FS, et al. Importância da orientação nutricional e do teor de fibras da dieta no controle glicêmico de pacientes diabéticos tipo 2 sob intervenção educacional intensiva. Arq bras endocrinol metab, São paulo, 2012, 56(2): 20-30.

9. DOSSE C, et al. Fatores Associados À Não Adesão Dos Pacientes Ao Tratamento De Hipertensão Arterial. Rev. Latino-Americana De Enfermagem, 2009, 17(2): 13-20.

10. FARIA HT, et al. Fatores Associados À Adesão Ao Tratamento De Pacientes Com Diabetes Mellitus. Escola De Enfermagem De Ribeirão Preto, Universidade De São Paulo, Ribeirão Preto, SP, Brasil. Universidade Federal Do Ceará, Fortaleza, CE, Brasil. 2013. Acta Paul Enferm. 2013; 26(3):231-7.

11. LADEIA VB. Fatores Que Influenciam Na Não Adesão Ao Tratamento Dos Usuários Portadores De Diabetes Mellitus. Curso De Especialização Em Saúde Da Família. Universidade Federal De Minas Gerais, Diamantina, 2013

12. LIMA TM, et al. Perfil De Adesão Ao Tratamento De Pacientes Atendidos Na Unidade Municipal De Saúde De Fátima Em Belém Pará Amazônia Brasil Rev Pan-Amaz Saude, 2010, 1(2): 113-120.

13. MACIEL EAM. A Não Adesão Ao Tratamento Da Hipertensão Por Pacientes Trabalhadores. Curso De Especialização Em Saúde Da Família. Universidade Federal De Minas Gerais, Brumadinho, 2012.

14. MILECH A. Diretrizes Da Sociedade Brasileira De Diabetes (2015-2016) / Organização José Egidio Paulo De Oliveira, Sérgio Vencio - São Paulo: A.C. Farmacêutica, 2016.

15. SOCIEDADE BRASILEIRA DE CARDIOLOGIA. VI Diretrizes Brasileiras de Hipertensão Arterial. Rev Bras Hipertens, 2010.

16. OSHIRO ML. Fatores Para Não-Adesão Ao Programa De Controle Da Hipertensão Arterial Em Campo Grande. MS : Uma Estudo De Caso E Controle. Universidade De Brasília, 2007

17. REZENDE DS, et al. Adherence to foot self-care in diabetes mellitus patients. Rev Bras Enferm [Internet], 2015, 68(1):103-8.

18. SANTOS ZMSA, et al. Adesão Do Cliente Hipertenso Ao Tratamento: Análise Com Abordagem Interdisciplinar. Texto \& Contexto Enfermagem, 2009, 14(3): 332-340.

19. SILVA MCM, et al. Avaliação Do Conhecimento Dos Portadores De Diabetes Melittus Sobre A Importância Da Manutenção Dos Níveis Glicêmicos Para Prevenção Da Nefropatia Diabética. Rev. Saúde e biologia, 2013, 7(3): 20-26.

20. SOUZA TP, et al. Atenção Qualificada Nos Cuidados Em Hipertensão E Diabetes Na Estratégia De Saúde Da Família. Revista Da Universidade Vale Do Rio Verde, Três Corações, 2015, 13(1): 689-696. 